\title{
The Role of Multidrug Interactions in the Safety of Pharmacotherapy for Concomitant Parkinson's Disease and Arterial Hypertension in Poland
}

\author{
Anna Bitner ${ }^{1 *}$, Paweł Zalewski ${ }^{1}$, Julia L Newton ${ }^{2}$ and Jacek J Klawe ${ }^{1}$ \\ ${ }^{1}$ Department of Hygiene and Epidemiology, Faculty of Health Sciences Ludwik Rydygier Collegium Medicum in Bydgoszcz Nicolaus Copernicus University in Torun, \\ Poland
}

${ }^{2}$ Institute for Ageing and Health, The Medical School, Newcastle University, United Kingdom

\begin{abstract}
Purpose: Multidrug interactions are amongst to the most frequent problems of pharmacotherapy. Such potentially harmful interactions are likely to occur in Parkinson's disease (PD) patients treated with concomitant arterial hypertension. The aim of this study was to analyze the prevalence of interactions between selected antiparkinson and hypotensive agents.

Methods: The analysis included data on the pharmacotherapy of PD and arterial hypertension, obtained from 80 men and women, diagnosed with Hoehn and Yahr stage II and III. However, the table presented data refer to persondrugs ( $n=186)$, as some of the respondents were prescribed more than one antiparkinson and/or hypotensive agent.

Results: A total of $53(28.5 \%)$ person-interactions were documented in the study group, among them $20(10.8 \%)$ minor, $28(25.8 \%)$ moderate and $5(28.5 \%)$ major ones. The presence of interactions was documented in $37(46.3 \%)$ patients. The number of different interactions present in a single patient amounted to three $(n=3,3.8 \%)$, two $(n=10$, $12.5 \%)$, one $(n=24,30.0 \%)$

Conclusions: Currently, we lack any detailed guidelines regarding pharmacotherapy of arterial hypertension and selection of hypotensive agents for patients with PD. Achieving desired hypotensive effect and reduction of adverse events resulting from drug-to-drug interactions constitute prerequisites of efficacious hypotensive treatment in patients with $P D$.
\end{abstract}

Keywords: Parkinson's disease; Arterial hypertension; Drug-drug interactions; Orthostatic hypotension

\section{Introduction}

Two or more drugs given at the same time may interact. The interaction may be potentiation or antagonism of one drug by another, or occasionally some other effect. Drug interactions may be pharmacodynamic or pharmacokinetic. Pharmacodynamic interactions are interactions between drugs which have similar or antagonistic pharmacological effects or side-effects. Pharmacokinetic interactions occur when one drug alters the absorption, distribution, metabolism, or excretion of another, thus increasing or reducing the amount of drug available to produce its pharmacological effects. Multidrug interactions belong to the most frequent problems of pharmacotherapy. Such potentially harmful interactions are likely to occur in Parkinson's disease (PD) patients treated for concomitant arterial hypertension [1,2].

According to the World Health Organization (WHO) arterial hypertension constitutes one of the most prevalent human diseases. Alterations of the 24-hour arterial pressure profile have been observed in the population of Parkinson's disease patients, but compared to the general population, a drop off in the arterial pressure is observed in the morning hours, along with its nocturnal elevation. Furthermore, epidemiological data suggests that concomitant orthostatic hypotension and arterial hypertension are present in $50 \%$ of individuals with PD $[1,2]$.

Pharmacotherapy for arterial hypertension in patients with Parkinson's disease is more challenging than in hypertensive individuals without other comorbidities. Most hypotensive agents can exacerbate the signs of orthostatic hypotension or interact with antiparkinson drugs, thus significantly deteriorating quality of life in PD patients. Therefore, the co-existence of arterial hypertension, orthostatic hypotension and the issue of drug - drug interactions constitute a serious therapeutic problem and necessitate individualization of treatment [3].

However, we lack evidence-based guidelines on pharmacotherapy of arterial hypertension in patients with Parkinson's disease, that would take into consideration the issue of drug interactions. Untreated arterial hypertension may lead to left ventricular hypertrophy in PD patients.

The aim of this study was to analyze the prevalence of interactions between selected antiparkinson and hypotensive agents in Poland. The analysis was based on a survey of medication use, conducted among randomly chosen 80 patients with concomitant $\mathrm{PD}$ and arterial hypertension. This article presents that drug-drug interactions are one of the commonest causes of medication error and very important problem in Poland particularly in the elderly due to polytherapy. Clinicians working with patients with Parkinson's disease need to know the significance of these interactions in order to properly counsel patients and prevent therapeutic problem.

*Corresponding author: Anna Bitner, Department of Hygiene and Epidemiology Collegium Medicum in Bydgoszcz, Nicolaus Copernicus University in Toruń, M. Sklodowskiej-Curie 9, 85-094 Bydgoszcz, Poland, Fax: +48 52 585-35-89;Tel: +48 52 585-36-16; E-mail: aniab@doktorant.umk.pl

Received October 08, 2014; Accepted November 04, 2014; Published November 11, 2014

Citation: Bitner A, Zalewski P, Newton JL, Klawe JJ (2014) The Role of Multidrug Interactions in the Safety of Pharmacotherapy for Concomitant Parkinson's Disease and Arterial Hypertension in Poland. J Pharmacovigilance 2: 151. doi:10.4172/23296887.1000151

Copyright: ( 2014 Bitner A, et al. This is an open-access article distributed under the terms of the Creative Commons Attribution License, which permits unrestricted use, distribution, and reproduction in any medium, provided the original author and source are credited. 


\section{Material and Methods}

\section{Material}

80 men and women (mean age $77.725 \pm 9.964$ years, median 81 years), diagnosed with Hoehn and Yahr stage II and III Parkinson's disease with concomitant arterial hypertension and lacking other comorbidities, were enrolled. The exclusion criteria pertained to the presence of other comorbidities (Endocrine diseases, Heart Muscle Disease, depression, disordersof thecentralnervous system). We extracted data on use of chronic medications and relevant medical conditions. The study was based on medication lists collected from patients selected from the Neurology Outpatient Clinic of the Dr. J. Biziel Memorial University Hospital no. 2 and at the Outpatient Clinic

\begin{tabular}{|c|c|c|c|c|c|}
\hline Agent 1 & $\mathbf{n}$ & $\%$ & Agent 2 & $\mathbf{n}$ & $\%$ \\
\hline Levodopa, benserazide & 118 & 63.4 & Amlodipine & 23 & 12.4 \\
\hline Ropinirole hydrochloride & 16 & 8.6 & Ramipril & 22 & 11.8 \\
\hline Selegiline hydrochloride & 15 & 8.1 & Metoprolol succinate & 18 & 9.7 \\
\hline Biperiden lactate & 10 & 5.4 & Bisoprolol fumarate & 15 & 8.1 \\
\hline Piribedil & 9 & 4.8 & Enalapril maleate & 12 & 6.5 \\
\hline Carbidopa, levodopa & 7 & 3.8 & Furosemide & 12 & 6.5 \\
\hline Amantadine hydrochloride & 5 & 2.7 & Indapamide & 9 & 4.8 \\
\hline Amantadine sulfate & 4 & 2.2 & Captopril & 7 & 3.8 \\
\hline Cabergoline & 1 & 0.5 & Spironolactone & 5 & 2.7 \\
\hline \multirow[t]{30}{*}{ Pridinol mesilate } & 1 & 0.5 & Vinpocetine & 5 & 2.7 \\
\hline & & & Isosorbide mononitrate & 5 & 2.7 \\
\hline & & & Piracetam & 5 & 2.7 \\
\hline & & & Verapamil hydrochloride & 4 & 2.2 \\
\hline & & & Torasemide & 4 & 2.2 \\
\hline & & & Lisinopril & 3 & 1.6 \\
\hline & & & Potassium chloride & 3 & 1.6 \\
\hline & & & Losartan potassium & 3 & 1.6 \\
\hline & & & Acebutolol & 3 & 1.6 \\
\hline & & & Perindopril erbumine & 2 & 1.1 \\
\hline & & & Valsartan & 2 & 1.1 \\
\hline & & & Clopidogrel & 2 & 1.1 \\
\hline & & & Metoprolol tartrate & 2 & 1.1 \\
\hline & & & Pentoxifylline & 2 & 1.1 \\
\hline & & & Carvedilol & 2 & 1.1 \\
\hline & & & Simvastatin & 2 & 1.1 \\
\hline & & & Dry extract of Convallaria & 1 & 0.5 \\
\hline & & & $\begin{array}{c}\text { Amiloride, } \\
\text { hydrochlorothiazide }\end{array}$ & 1 & 0.5 \\
\hline & & & Acetylsalicylic acid & 1 & 0.5 \\
\hline & & & Atenolol & 1 & 0.5 \\
\hline & & & Quinapril & 1 & 0.5 \\
\hline & & & Chlorthalidone & 1 & 0.5 \\
\hline & & & Metoprolol tartrate & 1 & 0.5 \\
\hline & & & Diltiazem hydrochloride & 1 & 0.5 \\
\hline & & & Lacidipine & 1 & 0.5 \\
\hline & & & $\begin{array}{l}\text { Losartan potassium, } \\
\text { hydrochlorothiazide }\end{array}$ & 1 & 0.5 \\
\hline & & & Betahistine dihydrochloride & 1 & 0.5 \\
\hline & & & Acenocoumarol & 1 & 0.5 \\
\hline & & & Perindopril, indapamide & 1 & 0.5 \\
\hline & & & Metoprolol & 1 & 0.5 \\
\hline
\end{tabular}

Agent 1: List of antiparkinson agents

Agent 2: List of hypotensive/cardiovascular agents

n: person-drugs

$\%$ : percentages refer to person-drugs $(n=186)$

Table 1: List of antiparkinson and hypotensive/cardiovascular agents prescribed to the study participants, percentages refer to person-drugs $(n=186)$. of Parkinsonism and Extrapyramidal Disorders of the Sue Ryder Home in Bydgoszcz, as well as the members of the Society of Individuals with Disabilities "Akson" and regional branch of the Kuyavian-Pomeranian Association of Individuals with Parkinson's Disease. Data from the medication lists was linked to the Polish interaction database. The examination included an evaluation of all medications in terms of occurring drug - drug interactions between antiparkinson and hypotensive agents (research questionnaire).

The respondents formed a homogenous group selected based on the United Kingdom Parkinson's Disease Society Brain Bank criteria. All patients satisfied at least three of the following criteria of Parkinson's disease: unilateral onset, presence of rest tremor with a $4-6 \mathrm{~Hz}$ frequency, progressive character, persistent asymmetry of symptoms, excellent $(70-100 \%)$ response to levodopa, severe levodopa-induced chorea, levodopa response for 5 years or more, clinical course of 10 years or more.

Concomitant arterial hypertension was the principal inclusion criterion of the study. The exclusion criteria pertained to the presence of other comorbidities, especially those precluding the diagnosis of Parkinson's disease, namely negative response to large doses of levodopa in the absence of malabsorption, history of definite encephalitis, history of repeated head injury, neuroleptic treatment at onset of symptoms, strictly unilateral features after 3 years, cerebellar signs, Babinski sign, presence of cerebral tumor, documented history of MPTP (1-methylo4-phenyl-1,2,3,6-tetrahydropyridine) exposure, lack of coordinated binocular vertical eye movement, and more than one affected relative.

The study was approved by the Human Research Committee of the Nicolas Copernicus University in Torun.

\section{Methods}

We used the "Drug interactions" database affiliated with the Ministry of Health, which allows for the identification of interactions between compared active compounds. The database was developed in cooperation with researchers from the Faculty of Pharmacology, Collegium Medicum at Jagiellonian University in Cracow. The database is updated on a monthly basis and all data is consistent with current legislation.

Program "Drug interactions" allows analysing the potential interactions between antihypertensive and anti-Parkinsonian agents and determining the degree of the clinical gravity drug-drug interactions (no interactions, non - significant interactions, significant interactions, very significant interactions).

Information about interactions we additionally supplemented for data from the BNF - a joint publication of the British Medical Association and the Royal Pharmaceutical Society of Great Britain. In analysis we also used data from: Micromedex ${ }^{\circ}$, Cerner Multum ${ }^{\mathrm{Tn}}$, Wolters Kluwer ${ }^{\mathrm{ns}}$, Lexicomp ${ }^{\oplus}$ and Stockley's ${ }^{\oplus}$.

\section{Statistical analysis}

The analysis included data on the pharmacotherapy of Parkinson's disease and arterial hypertension, obtained from 80 patients. However, the data refer to person-drugs ( $n=186)$, as some of the respondents were prescribed more than one antiparkinson and/or hypotensive agent.

The frequency of a drug/drug combination use and the prevalence of interactions of various grades are presented as numbers and percentages. The probability of an interaction between a pair antiparkinson agent + hypotensive/cardiovascular agent was determined with the univariate model of logistic regression. The odds ratio (OR) for using a given 
agent in a patient presenting with a drug-to-drug interaction was calculated, along with its 95\% confidence interval [between (-)95\%CI and $(+) 95 \% \mathrm{CI}]$. All statistical calculations were carried out with Statistica 10 software (StatSoft, Tulsa OK, United States), with the level of statistical significance set at $\mathrm{p} \leq 0.05$.

\section{Results}

Levodopa, benserazide $(\mathrm{n}=118,63.4 \%)$, ropinirole hydrochloride $(\mathrm{n}=16,8.6 \%)$, selegiline hydrochloride $(\mathrm{n}=15,8.1 \%)$, biperiden lactate $(\mathrm{n}=10,5.4 \%)$ and piribedil $(\mathrm{n}=9,4.8 \%)$ were the most frequently prescribed antiparkinson agents in the study group (Table 1).

Amlodipine ( $\mathrm{n}=23,12.4 \%)$, ramipril $(\mathrm{n}=22,11.8 \%)$, metoprolol succinate $(n=18,9.7 \%)$, bisoprololfumarate $(n=15,8.1 \%)$, enalapril maleate $(n=12,6.5 \%)$, furosemide $(n=12,6.5 \%)$ and indapamide $(n=9$, $4.8 \%$ ) were the hypotensive/cardiovascular agents used most frequently among our participants (Table 1).

The most prevalent combinations of agents from the two abovementioned groups included: levodopa, benserazide + ramipril $(\mathrm{n}=16,8.6 \%)$, levodopa, benserazide + amlodipine $(\mathrm{n}=15,8.1 \%)$, levodopa, benserazide + bisoprololfumarate $(n=10,5.4 \%)$, levodopa, benserazide + enalapril maleate $(n=10,5.4 \%)$ and levodopa, benserazide + metoprolol succinate ( $\mathrm{n}=8,4.3 \%$; Table 2$)$.

A total of $53(28.5 \%)$ person-interactions were documented in the

\begin{tabular}{|c|c|c|c|c|c|}
\hline Combination & $\mathbf{n}$ & $\%$ & Combination & $\mathbf{n}$ & $\%$ \\
\hline Levodopa, benserazide + Ramipril & 16 & 8.6 & Selegiline hydrochloride + Isosorbide mononitrate & 1 & 0.5 \\
\hline Levodopa, benserazide + Amlodipine & 15 & 8.1 & Piribedil + Bisoprolol fumarate & 1 & 0.5 \\
\hline Levodopa, benserazide + Bisoprolol fumarate & 10 & 5.4 & Levodopa, benserazide + Dry extract of Convallaria & 1 & 0.5 \\
\hline Levodopa, benserazide + Enalapril maleate & 10 & 5.4 & Levodopa, benserazide + Clopidogrel & 1 & 0.5 \\
\hline Levodopa, benserazide + Metoprolol succinate & 8 & 4.3 & Amantadine hydrochloride + Clopidogrel & 1 & 0.5 \\
\hline Levodopa, benserazide + Furosemide & 5 & 2.7 & Levodopa, benserazide + Amiloride, hydrochlorothiazide & 1 & 0.5 \\
\hline Selegiline hydrochloride + Metoprolol succinate & 4 & 2.2 & Biperiden lactate + Amlodipine & 1 & 0.5 \\
\hline Levodopa, benserazide + Isosorbide mononitrate & 4 & 2.2 & Ropinirole hydrochloride + Enalapril maleate & 1 & 0.5 \\
\hline Levodopa, benserazide + Piracetam & 4 & 2.2 & Amantadine hydrochloride + Bisoprolol fumarate & 1 & 0.5 \\
\hline Ropinirole hydrochloride + Amlodipine & 4 & 2.2 & Levodopa, benserazide + Acetylsalicylic acid & 1 & 0.5 \\
\hline Levodopa, benserazide + Indapamide & 4 & 2.2 & Levodopa, benserazide + Atenolol & 1 & 0.5 \\
\hline Levodopa, benserazide + Spironolactone & 3 & 1.6 & Levodopa, benserazide + Quinapril & 1 & 0.5 \\
\hline Levodopa, benserazide + Torasemide & 3 & 1.6 & Levodopa, benserazide + Chlorthalidone & 1 & 0.5 \\
\hline Levodopa, benserazide + Vinpocetine & 3 & 1.6 & Levodopa, benserazide + Metoprolol tartrate & 1 & 0.5 \\
\hline Levodopa, benserazide + Potassium chloride & 3 & 1.6 & Levodopa, benserazide + Diltiazem hydrochloride & 1 & 0.5 \\
\hline Levodopa, benserazide + Captopril & 3 & 1.6 & Amantadine hydrochloride + Ramipril & 1 & 0.5 \\
\hline Selegiline hydrochloride + Ramipril & 2 & 1.1 & Piribedil + Amlodipine & 1 & 0.5 \\
\hline Piribedil + Furosemide & 2 & 1.1 & Levodopa, benserazide + Lacidipine & 1 & 0.5 \\
\hline Levodopa, benserazide + Lisinopril & 2 & 1.1 & Levodopa, benserazide + Losartan potassium, hydrochlorothiazide & 1 & 0.5 \\
\hline Levodopa, benserazide + Metoprolol tartrate & 2 & 1.1 & Levodopa, benserazide + Betahistine dihydrochloride & 1 & 0.5 \\
\hline Levodopa, benserazide + Pentoxifylline & 2 & 1.1 & Levodopa, benserazide + Acenocoumarol & 1 & 0.5 \\
\hline Levodopa, benserazide + Carvedilol & 2 & 1.1 & Levodopa, benserazide + Simvastatin & 1 & 0.5 \\
\hline Amantadine hydrochloride + Metoprolol succinate & 2 & 1.1 & Piribedil + Acebutolol & 1 & 0.5 \\
\hline Piribedil + Indapamide & 2 & 1.1 & Biperiden lactate + Acebutolol & 1 & 0.5 \\
\hline Levodopa, benserazide + Losartan potassium & 2 & 1.1 & Carbidopa, levodopa + Acebutolol & 1 & 0.5 \\
\hline Biperiden lactate + Indapamide & 2 & 1.1 & Carbidopa, levodopa + Indapamide & 1 & 0.5 \\
\hline Ropinirole hydrochloride + Furosemide & 2 & 1.1 & Cabergoline + Furosemide & 1 & 0.5 \\
\hline Biperiden lactate + Metoprolol succinate & 2 & 1.1 & Biperiden lactate + Enalapril maleate & 1 & 0.5 \\
\hline Ropinirole hydrochloride + Bisoprolol fumarate & 2 & 1.1 & Biperiden lactate + Furosemide & 1 & 0.5 \\
\hline Ropinirole hydrochloride + Ramipril & 2 & 1.1 & Biperiden lactate + Verapamil hydrochloride & 1 & 0.5 \\
\hline Ropinirole hydrochloride + Captopril & 2 & 1.1 & Piribedil + Verapamil hydrochloride & 1 & 0.5 \\
\hline Levodopa, benserazide + Perindopril erbumine & 1 & 0.5 & Pridinol mesilate + Amlodipine & 1 & 0.5 \\
\hline Levodopa, benserazide + Verapamil hydrochloride & 1 & 0.5 & Amantadine sulfate + Perindopril, indapamide & 1 & 0.5 \\
\hline Levodopa, benserazide + Valsartan & 1 & 0.5 & Ropinirole hydrochloride + Simvastatin & 1 & 0.5 \\
\hline Selegiline hydrochloride + Perindopril erbumine & 1 & 0.5 & Carbidopa, levodopa + Amlodipine & 1 & 0.5 \\
\hline Selegiline hydrochloride + Verapamil hydrochloride & 1 & 0.5 & Carbidopa, levodopa + Piracetam & 1 & 0.5 \\
\hline Selegiline hydrochloride + Valsartan & 1 & 0.5 & Piribedil + Vinpocetine & 1 & 0.5 \\
\hline Selegiline hydrochloride + Bisoprolol fumarate & 1 & 0.5 & Carbidopa, levodopa + Metoprolol & 1 & 0.5 \\
\hline Selegiline hydrochloride + Spironolactone & 1 & 0.5 & Carbidopa, levodopa + Furosemide & 1 & 0.5 \\
\hline Selegiline hydrochloride + Torasemide & 1 & 0.5 & Carbidopa, levodopa + Spironolactone & 1 & 0.5 \\
\hline Selegiline hydrochloride + Vinpocetine & 1 & 0.5 & Amantadine sulfate + Ramipril & 1 & 0.5 \\
\hline Ropinirole hydrochloride + Metoprolol succinate & 1 & 0.5 & Amantadine sulfate + Captopril & 1 & 0.5 \\
\hline Ropinirole hydrochloride + Losartan potassium & 1 & 0.5 & Selegiline hydrochloride + Captopril & 1 & 0.5 \\
\hline Amantadine sulfate + Metoprolol succinate & 1 & 0.5 & Biperiden lactate + Lisinopril & 1 & 0.5 \\
\hline
\end{tabular}

n: person-drugs

$\%$ : percentages refer to person-drugs $(n=186)$

Table 2: Combinations of antiparkinson + hypotensive/cardiovascular agents prescribed to the study participants, percentages refer to person-drugs ( $\mathrm{n}=186)$ 


\begin{tabular}{|c|c|c|c|c|c|c|c|c|}
\hline Agent 1 & $\mathbf{n}$ & $\%$ & Agent 2 & $\mathbf{n}$ & $\%$ & Combination & $\mathbf{n}$ & $\%$ \\
\hline Levodopa, benserazide & 35 & 66.0 & Amlodipine & 20 & 37.7 & Levodopa, benserazide + Amlodipine & 15 & 28.3 \\
\hline Ropinirole hydrochloride & 7 & 13.2 & Indapamide & 7 & 13.2 & Levodopa, benserazide + Furosemide & 5 & 9.4 \\
\hline Selegiline hydrochloride & 5 & 9.4 & Furosemide & 6 & 11.3 & Selegiline hydrochloride + Metoprolol succinate & 4 & 7.5 \\
\hline Carbidopa, levodopa & 4 & 7.5 & Metoprolol succinate & 5 & 9.4 & Ropinirole hydrochloride + Amlodipine & 4 & 7.5 \\
\hline \multirow[t]{16}{*}{ Biperiden lactate } & 2 & 3.8 & Spironolactone & 4 & 7.5 & Levodopa, benserazide + Indapamide & 4 & 7.5 \\
\hline & & & Bisoprolol fumarate & 3 & 5.7 & Levodopa, benserazide + Spironolactone & 3 & 5.7 \\
\hline & & & Torasemide & 3 & 5.7 & Levodopa, benserazide + Torasemide & 3 & 5.7 \\
\hline & & & Perindopril erbumine & 1 & 1.9 & Biperiden lactate + Indapamide & 2 & 3.8 \\
\hline & & & Verapamil hydrochloride & 1 & 1.9 & Ropinirole hydrochloride + Bisoprolol fumarate & 2 & 3.8 \\
\hline & & & Amiloride, hydrochlorothiazide & 1 & 1.9 & Levodopa, benserazide + Perindopril erbumine & 1 & 1.9 \\
\hline & & & Chlorthalidone & 1 & 1.9 & Levodopa, benserazide + Verapamil hydrochloride & 1 & 1.9 \\
\hline & & & Diltiazem hydrochloride & 1 & 1.9 & Selegiline hydrochloride + Bisoprolol fumarate & 1 & 1.9 \\
\hline & & & & & & Levodopa, benserazide + Amiloride, hydrochlorothiazide & 1 & 1.9 \\
\hline & & & & & & Levodopa, benserazide + Chlorthalidone & 1 & 1.9 \\
\hline & & & & & & Levodopa, benserazide + Diltiazem hydrochloride & 1 & 1.9 \\
\hline & & & & & & Carbidopa, levodopa + Indapamide & 1 & 1.9 \\
\hline & & & & & & Carbidopa, levodopa + Amlodipine & 1 & 1.9 \\
\hline & & & & & & Carbidopa, levodopa + Furosemide & 1 & 1.9 \\
\hline & & & & & & Carbidopa, levodopa + Spironolactone & 1 & 1.9 \\
\hline & & & & & & Ropinirole hydrochloride + Metoprolol succinate & 1 & 1.9 \\
\hline
\end{tabular}

Agent 1: List of antiparkinson agents

Agent 2: List of hypotensive/cardiovascular agents

n: person-interactions

$\%$ : percentages refer to person-interactions $(n=53)$

Table 3: List of antiparkinson and hypotensive/cardiovascular agents and the combinations of them that were involved in interactions of any grade, percentages refer to person-interactions $(n=53)$

\begin{tabular}{|c|c|c|c|c|c|c|c|c|}
\hline Agent 1 & $\mathbf{n}$ & $\%$ & Agent 2 & $\mathbf{n}$ & $\%$ & Combination & $\mathbf{n}$ & $\%$ \\
\hline Levodopa, benserazide & 18 & 54.5 & Furosemide & 6 & 18.2 & Levodopa, benserazide + Furosemide & 5 & 15.2 \\
\hline Ropinirole hydrochloride & 7 & 21.2 & Metoprolol succinate & 5 & 15.2 & Selegiline hydrochloride + Metoprolol succinate & 4 & 12.1 \\
\hline Selegiline hydrochloride & 5 & 15.2 & Indapamide & 5 & 15.2 & Ropinirole hydrochloride + Amlodipine & 4 & 12.1 \\
\hline Levodopa, carbidopa & 3 & 9.1 & Spironolactone & 4 & 12.1 & Levodopa, benserazide + Indapamide & 4 & 12.1 \\
\hline & & & Amlodipine & 4 & 12.1 & Levodopa, benserazide + Spironolactone & 3 & 9.1 \\
\hline & & & Bisoprolol fumarate & 3 & 9.1 & Levodopa, benserazide + Torasemide & 3 & 9.1 \\
\hline & & & Torasemide & 3 & 9.1 & Ropinirole hydrochloride + Bisoprolol fumarate & 2 & 6.1 \\
\hline & & & Perindopril erbumine & 1 & 3.0 & Levodopa, benserazide + Perindopril erbumine & 1 & 3.0 \\
\hline & & & Amiloride, hydrochlorothiazide & 1 & 3.0 & Selegiline hydrochloride + Bisoprolol fumarate & 1 & 3.0 \\
\hline & & & Chlorthalidone & 1 & 3.0 & Levodopa, benserazide + Amiloride, hydrochlorothiazide & 1 & 3.0 \\
\hline & & & & & & Levodopa, benserazide + Chlorthalidone & 1 & 3.0 \\
\hline & & & & & & Carbidopa, levodopa + Indapamide & 1 & 3.0 \\
\hline & & & & & & Carbidopa, levodopa + Furosemide & 1 & 3.0 \\
\hline & & & & & & Carbidopa, levodopa + Spironolactone & 1 & 3.0 \\
\hline & & & & & & Ropinirole hydrochloride + Metoprolol succinate & 1 & 3.0 \\
\hline
\end{tabular}

Agent 1: List of antiparkinson agents

Agent 2: List of hypotensive/cardiovascular agents

n: person-interactions

$\%$ : percentages refer to person-interactions $(n=33)$

Table 4: List of antiparkinson and hypotensive/cardiovascular agents and the combinations of them, that were involved in major/moderate interactions, percentages refer to person-interactions $(n=33)$.

study group, among them $20(10.8 \%)$ minor, 28 (25.8\%) moderate and $5(28.5 \%)$ major ones. The presence of interactions was documented in $37(46.3 \%)$ patients. The number of different interactions present in a single patient amounted to three $(\mathrm{n}=3,3.8 \%)$, two $(\mathrm{n}=10,12.5 \%)$ and one $(n=24,30.0 \%)$. None of the patients presented with more than one major interaction. Among the subjects with three different interactions, there was one individual with two moderate and one major interaction. The group of persons with two different interactions included two patients who presented solely with moderate interactions.

Levodopa, benserazide were the antiparkinson agent that was most frequently involved in interactions of any grade $(n=35,66.0 \%$; Table 3$)$.
In turn, amlodipine was the hypotensive agent most often being involved in interactions of any grade $(n=20,37.7 \%$; Table 3$)$. Consequently, the interaction levodopa, benserazide + amlodipine proved to be the most prevalent interaction of any grade in our group $(n=15,28.3 \%$; Table 3$)$.

Selegiline hydrochloride was the only antiparkinson agent involved solely in major interactions $(n=5,100.0 \%)$. The group of hypotensive agents that were involved solely in major interactions included metoprolol succinate $(n=4,80.0 \%)$ and bisoprololfumarate $(n=1$, $20.0 \%)$.

Levodopa, benserazide were the antiparkinson agent that was most frequently involved in major/moderate interactions $(n=18,54.6 \%$; 


\begin{tabular}{|c|c|c|c|c|c|c|c|c|c|}
\hline Agent 1 & OR & $(-) 95 \% \mathrm{Cl}$ & (+) $95 \% \mathrm{Cl}$ & p & Agent 2 & OR & $(-) 95 \% \mathrm{Cl}$ & (+) $95 \% \mathrm{Cl}$ & $\mathbf{p}$ \\
\hline Amlodipine & 26.3 & 7.3 & 94.5 & 0.000 & Levodopa, benserazide & 1.2 & 0.6 & 2.3 & 0.643 \\
\hline Indapamide & 10.0 & 2.0 & 50.2 & 0.005 & Ropinirole hydrochloride & 2.1 & 0.7 & 6.0 & 0.165 \\
\hline Furosemide & 2.7 & 0.8 & 8.9 & 0.099 & Selegiline hydrochloride & 1.3 & 0.4 & 4.0 & 0.666 \\
\hline Metoprolol succinate & 1.0 & 0.3 & 2.9 & 0.944 & Carbidopa, levodopa & 3.5 & 0.8 & 16.5 & 0.106 \\
\hline Spironolactone & 10.8 & 1.2 & 100.3 & 0.035 & Biperiden lactate & 0.6 & 0.1 & 3.0 & 0.544 \\
\hline Bisoprolol fumarate & 0.6 & 0.2 & 2.3 & 0.451 & & & & & \\
\hline Torasemide & 7.9 & 0.8 & 79.1 & 0.076 & & & & & \\
\hline
\end{tabular}

Agent 1: List of hypotensive/cardiovascular agents

Agent 2: List of antiparkinson agents

p: $p$-value, probability value

Table 5: Odds ratios (ORs) for using a given antiparkinson and hypotensive/cardiovascular agent in a patient presenting with an interaction of any grade.

Table 4). In turn, furosemide was the hypotensive/cardiovascular agent most often being involved in the major/moderate interactions $(n=6$, 18.2\%;Table 4). Therefore, the combination levodopa, benserazide + furosemide was the most prevalent major/moderate interaction documented in our group $(n=5,15.2 \%$; Table 4$)$.

The use of any antiparkinson agents was not associated with increased probability of interaction (Table 5). In turn, the probability of interaction was significantly (up to 26 times) higher in patients who were prescribed amlodipine, spironolactone or indapamide (Table 5).

\section{Discussion}

Administration of combined preparations of levodopa and peripheral decarboxylase inhibitors (benserazide/carbidopa) is postulated to be the most efficient therapeutic option for Parkinson's disease, and for more than 30 years constitutes the gold standard in treatment of this condition. Early implementation of levodopa can probably be reflected by a 5-7 year increase in life expectancy of PD patients [4].

Unfortunately, the combinations of levodopa and benserazide can interact with some hypotensive agents. We identified the combination levodopa, benserazide + furosemide as the most prevalent of major/ moderate multidrug interactions in our group.

While the use of any of the antiparkinson agents was not associated with a significant increase in the interaction risk, the latter increased significantly (up to 26 times) in the case of amlodipine, spironolactone or indapamide administration.

Therefore, thiazide and thiazide-like diuretics (e.g. hydrochlorothiazide, indapamide), loop diuretics (e.g. furosemide, torasemide) and potassium-sparing diuretics (e.g. spironolactone) should be prescribed carefully to patients being treated with combinations of levodopa and benserazide, as the latter agents may enhance the hypotensive effect of diuretics and thus promote orthostatic hypotension [5].

Orthostatic hypotension is the most frequent cardiovascular disorder observed in Parkinson's disease patients. Usually this condition is defined as at least a $20 \mathrm{~mm} \mathrm{Hg}$ decrease in systolic blood pressure during initial three minutes of verticalization. The main signs of orthostatic hypotension include vertigo, visual disturbances, neck pain in a standing position and, less often, the signs of myocardial ischemia. Vertigo experienced shortly after standing up constitutes a frequent cause of falls. Frequent prevalence of this symptom, resulting from the presence of drug-to-drug interactions, may significantly diminish the quality of life of PD patients $[5,6]$.

Our analysis revealed that a combination of levodopa and benserazide with amlodipine was the most frequent multidrug interaction of any grade present in our respondents. Concomitant administration of calcium channel blockers (other than lacidipine) and combination of levodopa with benserazide may lead to a minor pharmacokinetic interaction. The interaction results from the impairment of synaptic transmission by the calcium channel blockers and resultant attenuation of therapeutic effects of levodopa and benserazide.

Other, most commonly prescribed combinations of antiparkinson and hypotensive agents in our group were levodopa, benserazide + bisoprololfumarate/succinate and levodopa, benserazide + enalapril maleate.

Angiotensin converting enzyme inhibitors (ACE-I), such as enalapril maleate, are not involved in moderate or major interactions with levodopa and benserazide used in the treatment of Parkinson's disease. Although the angiotensin converting enzyme inhibitors are tolerated well by most of the patients, their administration may be associated with some adverse effects, such as orthostatic hypotension, dry cough, hyperkalemia, acute renal failure, proteinuria, and angioedema [7].

Since orthostatic hypotension constitutes the most frequent cardiovascular problem in PD patients, one should avoid further increase in its risk associated with administration of ACE-I. The signs of hypotension observed after administration of angiotensin converting enzyme inhibitors can result from the lack of angiotensin II-induced increase in vascular resistance. This effect is particularly evident in individuals with elevated plasma activity of renin, especially after the first dose of ACE-I [7].

Our survey did not reveal any interactions between betaadrenoreceptor antagonists (i.e. bisoprololfumarate/succinate) and levodopa preparations. Nevertheless, one should be aware of potential adverse effects of the abovementioned hypotensive agents on the central nervous system, namely fatigue, headache, sleep disorders, sleeplessness, "vivid dreams" and depression. Moreover, the betaadrenoreceptor antagonists can induce or exacerbate impotency and cause a loss of libido in some patients. As the latter symptoms are also characteristic for Parkinson's disease, their exacerbation during hypotensive treatment constitutes indication to discontinuing the betaadrenoreceptor antagonists. However, it should be remembered that rapid discontinuation of beta blockers after their chronic use can cause "withdrawal syndromes", such as arrhythmia, arterial hypertension and exacerbation of the ischemic heart disease [8].

Selegiline hydrochloride turned out to be the only antiparkinson agent that was involved in major drug-to-drug interactions in our respondents. Concomitant therapy with beta-blockers and selegiline leads to a major interaction that is associated with the risk of bradycardia and life-threatening adrenergic storm. 
We documented the presence of multidrug interactions in as many as $37(46.3 \%)$ surveyed patients. The exact prevalence of drug-to-drug interactions among patients with concomitant Parkinson's disease and arterial hypertension was not a subject of any controlled epidemiological studies,probably because of under-reporting of such events to the respective centers. Since alterations of drug pharmacokinetics can be avoided with the help of good knowledge of pharmacotherapy, one should consider the issue of potential drug-to-drug interactions during selection of a therapeutic strategy.

\section{Conclusions}

This study reports upon drug interactions between antiparkinsonian medications and antihypertensive drugs in $\mathrm{PD}$ Parkinson's disease patients. It categorizes adverse effects, most notably orthostatic hypotension, to derive correlations between classes of drugs and severity of adverse effects.

Currently, we lack any detailed guidelines regarding pharmacotherapy of arterial hypertension and selection of hypotensive agents for patients with Parkinson's disease. Achieving desired hypotensive effect and reduction of adverse events resulting from drugto-drug interactions constitute prerequisites of efficacious hypotensive treatment in patients with Parkinson's disease.

In view of the existing therapeutic guidelines for Parkinson's disease and arterial hypertension, and potential interactions between agents used in the treatment of these conditions, an individualized hypotensive therapy, adjusted for used antiparkinson agents, should be proposed to $\mathrm{PD}$ patients.

The results of previous clinical trials suggest that the antagonists of type 1 angiotensin II receptors $\left(\mathrm{AT}_{1}\right)$ are characterized by a low number of side effects, long-term hypotensive effect and mild onset of action. Consequently, the use of these agents seems to be an optimal option of hypotensive therapy for patients with concomitant Parkinson disease [9].

\section{Key Points}

- We lack detailed guidelines on the pharmacotherapy of arterial hypertension in patients with Parkinson's disease.

- Pharmacotherapy of arterial hypertension in this group should be individualized.
- Angiotensin-converting enzyme inhibitors, antagonists of $\mathrm{AT}_{1}$ receptor for angiotensin II or antagonists of $\beta$-adrenoreceptors seem to be the safest option of hypotensive treatment for patients with Parkinson's disease.

- Clinicians should feel reluctant to initiate antiparkinson treatment with medicines for hypertension (drug - drug interactions) in patients with Parkinson's disease, because such treatment worsens the quality of life of patients.

\section{Conflict of Interest}

The authors have no conflict of interest to report. All authors of this research paper have directly participated in the planning, execution, or analysis of this study. All authors of this paper have read and approved the final version submitted.

\section{References}

1. Tsukamoto T, Kitano Y, Kuno S (2013) Blood pressure fluctuation and hypertension in patients with Parkinson's disease. Brain Behav 3: 710-714.

2. Kaufmann H, Goldstein DS (2013) Autonomic dysfunction in Parkinson disease. HandbClinNeurol 117: 259-278.

3. Jain S, Goldstein DS (2012) Cardiovascular dysautonomia in Parkinson disease: from pathophysiology to pathogenesis. Neurobiol Dis $46: 572-580$.

4. Gasser UE, Fischer A, Timmermans JP, Arnet I (2012) Pharmaceutical quality of seven generic Levodopa/Benserazide products compared with original Madopar®/ Prolopa®. BMC PharmacolToxicol 14: 24.

5. Oka H, Yoshioka M, Onouchi K, Morita M, Mochio S, et al. (2007) Characteristics of orthostatic hypotension in Parkinson's disease. Brain. A journal of neurology 130: $2425-2432$.

6. Goldstein DS (2003) Dysautonomia in Parkinson's disease; neurocardiological abnormalities. Lancet Neurol 1: 669-676.

7. Sweitzer NK (2003) What Is an Angiotensin Converting Enzyme Inhibitor? Circulation 108: e16-e18.

8. Frishman WH (2003) Beta-Adrenergic Blockers. Circulation 107: e117-e119.

9. vanZwieten PA (2006) Angiotensin II receptor antagonists (AT,-blockers, ARBs, sartans): similarities and differences. Neth Heart J 14: 381-387. 